\title{
Cuidados com a pele adotados por gestantes assistidas em serviços públicos de saúde*
}

\author{
Skin Care adopted by pregnant women seen by public health services \\ Cuidados con la piel adoptados por gestantes atendidas en servicios públicos de salud
}

\author{
Maristela Belletti Mutt Urasaki ${ }^{1}$
}

\begin{abstract}
RESUMO
Objetivos: Descrever os cuidados com a pele adotados por mulheres no período gestacional e identificar a adequação das práticas utilizadas. Métodos: Estudo descritivo exploratório com participação de 124 gestantes atendidas em quatro unidades básicas de saúde da região Leste do Município de São Paulo. Resultados: A maioria das gestantes afirmou cuidar da pele (74,2\%), entretanto, constatou-se inadequação de várias práticas que podem contribuir no desencadeamento de alterações cutâneas; mais da metade das mulheres (56,5\%) afirmou não ter recebido orientações acerca dos cuidados com a pele. Conclusões: Os resultados evidenciaram a importância da inclusão da abordagem sobre os cuidados da pele nos programas de educação em saúde desenvolvidos em serviços que proporcionam atendimento à gestante.
\end{abstract}

Descritores: Dermatologia; Gravidez; Pele; Cuidados de enfermagem

\begin{abstract}
Objectives: To describe skin care practices used by women during pregnancy and to determine if these practices are adequate. Methods: A descriptive exploratory study with 124 pregnant women enrolled in four basic health units in the eastern region of São Paulo. Results: Most of the women affirmed that they practiced skin care regimens $(74.2 \%)$, however, several practices were found to be inadequate and could contribute to the onset of skin changes. More than half of these women $(56.5 \%)$ noted that they had received guidance about skin care. Conclusions: The results showed the importance of including information related to skin care when developing health education services for pregnant women.
\end{abstract}

Keywords: Dermatology; Pregnancy; Skin; Nursing care

\section{RESUMEN}

Objetivos: Describir los cuidados con la piel adoptados por mujeres en el período gestacional e identificar la adecuación de las prácticas utilizadas. Métodos: Estudio descriptivo exploratorio realizado con la participación de 124 gestantes atendidas en cuatro unidades básicas de salud de la región Este del Municipio de Sao Paulo. Resultados: La mayoría de las gestantes afirmó cuidar la piel (74,2\%), no obstante, se constató inadecuación de varias prácticas que pueden contribuir en la aparición de alteraciones cutáneas; más de la mitad de las mujeres $(56,5 \%)$ afirmó no haber recibido orientaciones acerca de los cuidados con la piel. Conclusiones: Los resultados evidenciaron la importancia de la inclusión de temas relativos al cuidado de la piel en los programas de educación en salud desarrollados en los servicios que proporcionan atención a la gestante.

Descriptores: Dermatologia; Embarazo; Piel; Cuidados de enfermería

\footnotetext{
* Este texto faz parte da pesquisa "Cuidados com a pele adotados por gestantes de um serviço público da região leste do município de São Paulo", desenvolvida pela autora e apresentada à Escola de Artes, Ciências e Humanidades da Universidade de São Paulo - USP - São Paulo (SP), Brasil. .

${ }^{1}$ Curso de Obstetrícia da Escola de Artes, Ciências e Humanidades, Universidade de São Paulo - USP - São Paulo (SP), Brasil.
} 


\section{INTRODUÇÃO}

Durante a gestação, ocorrem modificações que tornam a pele da mulher predisposta a ocorrências fisiológicas e patológicas, exigindo rigor nos cuidados, de modo que a forma e a função sejam preservadas.

Os achados dermatológicos mais frequentes no período gestacional são as alterações fisiológicas da pele, que são modificações e não são consideradas doenças pelos especialistas. As alterações pigmentares, do tecido conjuntivo (estrias), vasculares e dos pelos e unhas ${ }^{(1-5)}$ fazem parte desse grupo. Outros autores incluem também a acne ${ }^{(6)}$.

Dentre as alterações pigmentares, destacam-se as manchas que ocorrem em $75 \%$ das gestantes ${ }^{(7)}$ e a hiperpigmentação que se manisfesta em $90 \%$ delas $^{(8)}$. As estrias afetam $90 \%$ das grávidas ${ }^{(5)}$ e os microvasos atingem cerca de $40 \%{ }^{(4)}$. Por sua vez, a acne, é a principal causa de consulta dermatológica na população ${ }^{(9)}$, abrangendo também as gestantes. Estas podem desenvolver o quadro pela primeira vez, como ter o agravamento de uma acne preexistente.

Uma combinação de fatores está associada ao desenvolvimento e incremento das alterações dermatológicas apontadas, entre eles, predisposição genética, fatores hormonais e estilo de vida ${ }^{(3-4,6-7)}$. Este último componente agrega: sedentarismo, inadequação da alimentação e hidratação, exposição solar e cuidados tópicos.

O fato das alterações dermatológicas serem descritas como fisiológicas, não minimizam o desconforto sentido pelas mulheres ${ }^{(4)}$. As ocorrências podem ser esteticamente significativas e comprometer a dimensão biológica, psicoemocional e social da gestante. Estudos evidenciam que problemas dermatológicos, incluindo os de baixa morbidade, provocam impacto na qualidade de vida das pessoas ${ }^{(10-11)}$

Nesse sentido, a adoção de medidas relativamente simples pode contribuir para a saúde e bem estar. No período gestacional, o ideal é adotar cuidados preventivos e evitar procedimentos e produtos de maior eficácia frente aos riscos à mãe e ao feto ${ }^{(2,12-13)}$.

Diante do exposto, este estudo teve por objetivo descrever os cuidados com a pele adotados pela mulher no período gestacional e identificar a sua apropriação. Espera-se que este estudo contribua para despertar no profissional de saúde a necessidade de investimento educacional para problemas de baixa morbidade.

\section{MÉTODOS}

Trata-se de um estudo quantitativo, descritivo e exploratório realizado em quatro unidades básicas de saúde (UBS), pertencentes à Administração Regional da Penha, zona Leste do Município de São Paulo. O período de coleta de dados transcorreu entre os meses de março a julho de 2008.

Os sujeitos da pesquisa foram gestantes usuárias das unidades. Os critérios de inclusão adotados foram: ser maior de 18 anos, idade gestacional maior ou igual a 13 semanas e não apresentar problemas dermatológicos pré-existentes. O contato foi feito nas salas de espera das consultas de pré-natal, realizadas por enfermeiras e médicos, e a coleta foi realizada antes da consulta. A amostra estudada foi por conveniência, composta por 124 gestantes, decorrentes do tempo de coleta. Todas as gestantes que se encontravam presentes no local, no momento da coleta, foram convidadas a participar. Não houve recusa. Às gestantes, foi assegurado, anonimato quanto às informações prestadas. As participantes assinaram um Termo de Consentimento Livre e Esclarecido. A pesquisa foi aprovada pelo Comitê de Ética em Pesquisa da Secretaria Municipal de Saúde de São Paulo (CEP/SMS 301/07).

Para a coleta de dados, foi usado um formulário, preenchido pela pesquisadora e por uma aluna de graduação treinada. $\mathrm{O}$ instrumento compôs-se de cinco domínios: caracterização da população, estilo de vida, cuidados com a pele e cabelos, autopercepção sobre cuidados com a pele e orientações recebidas por profissionais. As variáveis consideradas para estilo de vida foram: permanência prolongada na posição de pé, prática de atividade física programada, freqüência da atividade, exposição solar regular no período das 10 às 16 horas, tempo de exposição solar, alimentação e hidratação. As variáveis consideradas para cuidados com a pele e o cabelo foram: frequência da higiene corporal, temperatura da água no banho e tempo gasto no banho em minutos, tipo de sabonete, método de uso e áreas de uso direto, frequência da higiene dos cabelos, tipo do produto utilizado, hidratação tópica facial e corporal, tipo de produto usado, frequência do uso do protetor solar, fator utilizado e controle de peso. Para conhecer a temperatura da água no banho, foi usada uma escala de quatro níveis e aceita a menção dada pela gestante. Quanto ao método do uso de sabonetes, foram considerados o uso de esponjas, buchas e espuma.

Os dados coletados foram trabalhados e analisados utilizando-se a planilha eletrônica Excel (Microsoft).

\section{RESULTADOS}

As gestantes que compuseram a amostra tinham entre 18 e 41 anos, sendo a média de idade 26,5 anos. A escolaridade mais frequente foi o ensino médio completo, representada por $33 \%$ das gestantes. Apenas uma $(0,8 \%)$ não frequentou a escola. Quanto à renda, $76(63,3 \%)$ respondentes mencionaram receber menos de três salários mínimos. A idade gestacional ficou 
distribuída em: 57 (46\%) mulheres no segundo trimestre e $67(54 \%)$ no terceiro trimestre.

Os resultados sobre comportamento revelaram que do total de gestantes $62(50 \%)$ afirmaram não permanecer mais que uma hora na posição em pé, outras $62(50 \%)$ disseram manter atividade que exigia permanência em pé prolongada. Dessas, $11(17 \%)$ mencionaram entre uma a duas horas ao dia, $17(27,4 \%)$ de duas a quatro horas, $16(25,8)$ de quatro a seis horas e $18(29 \%)$ mais de seis horas ao dia.

Quanto à prática de atividade física programada, verificou-se que a grande maioria, 107 (86,3\%), não realizava qualquer tipo de exercício físico e a minoria $17(13,7 \%)$ o faz; dessas 15 (88,2\%) mulheres citaram caminhada, uma $(5,9 \%)$ natação e uma $(5,9 \%)$ o uso de bicicleta. A frequência da atividade física ficou distribuída em: $6(35,3 \%)$ praticavam duas vezes por semana, duas $(11,8 \%)$ três vezes por semana, $3(17,6 \%)$ cinco vezes por semana, uma $(5,9 \%)$ seis vezes e $5(29,4 \%)$ sete vezes por semana.

Os dados relativos à exposição solar regular durante o horário das 10 às 16 horas, o local e o tempo de exposição em 24 horas estão apresentados na Tabela 1.

Tabela 1 - Gestantes segundo exposição regular à radiação solar e tempo de exposição. São Paulo, março a julho de 2008.

\begin{tabular}{lrr}
\hline Exposição regular das $\mathbf{1 0}$ às $\mathbf{1 6} \mathbf{h}$ & $\mathbf{n}$ & $\mathbf{\%}$ \\
\hline Não permaneceu ex posta & 65 & 52,4 \\
Exposição no domicílio & 23 & 18,5 \\
Exposção no lazer & 15 & 12,1 \\
Expo sição no trabalho & 20 & 16,1 \\
Expo sção na atividade es portiva & 1 & 0,8 \\
\hline Total & 124 & 100,0 \\
\hline Tempo de exposição solar em $\mathbf{2 4} \mathbf{h}$ & $\mathbf{n}$ & $\mathbf{\%}$ \\
\hline Menos de 1 h & 53 & 42,7 \\
1h & 31 & 25,0 \\
2h & 19 & 15,3 \\
3h & 10 & 8,1 \\
4h & 7 & 5,6 \\
6h & 4 & 3,2 \\
\hline Total & 124 & 100,0 \\
\hline
\end{tabular}

A respeito dos cuidados diretos com a pele e cabelo, foram investigadas a frequência da higiene corporal e a temperatura da água, durante inverno e verão (Tabela 2).

Quanto ao tempo gasto no banho, $47(37,9 \%)$ gestantes informaram permanecer mais de 15 minutos no chuveiro, $36(29 \%)$ de 6 a 10 minutos, $22(17,8 \%)$ de 11 a 15 minutos, $14(11,3 \%)$ de 3 a 5 minutos e $5(4 \%)$ não souberam precisar. $\mathrm{O}$ tipo de sabonete usado pelas gestantes e o método de aplicação estão apresentados na Tabela 3.

Das gestantes que usaram sabonete direto sobre a pele, $80(92,6 \%)$ mencionaram usar em todas as áreas do corpo. Outras 18 (22\%) citaram usar em algumas áreas. Região axilar foi citada por $14(17,1 \%)$, genitais
$13(15,9 \%)$, membros por $8(9,8 \%)$, pescoço e face por $4(4,9 \%)$, respectivamente, abdome por $5(6,1 \%)$ e mão por uma $(1,2 \%)$.

Tabela 2 - Gestantes, segundo frequência do banho e temperatura da água. São Paulo, março a julho de 2008.

\begin{tabular}{lrrrr}
\hline \multirow{2}{*}{ Frequência do banho } & \multicolumn{2}{c}{ Inverno } & \multicolumn{2}{c}{ Verão } \\
\cline { 2 - 5 } & $\mathbf{n}$ & $\mathbf{\%}$ & $\mathbf{n}$ & $\mathbf{0}$ \\
\hline 1x dia & 56 & 45,2 & 26 & 21,0 \\
2x dia & 62 & 50,0 & 75 & 60,5 \\
3x dia & 3 & 2,4 & 22 & 17,7 \\
4x dia & - & - & 1 & 0,8 \\
Dias alternados & 3 & 2,4 & - & - \\
\hline Total & 124 & 100,0 & 124 & 100,0 \\
\hline Temperatura da água & $\mathbf{n}$ & $\mathbf{0}$ & $\mathbf{n}$ & $\mathbf{\%}$ \\
\hline Fria & - & - & 12 & 9,7 \\
Morna & 34 & 27,4 & 73 & 58,9 \\
Quente & 59 & 47,6 & 29 & 23,4 \\
Muito quente & 31 & 25,0 & 10 & 8,1 \\
\hline Total & 124 & 100,0 & 124 & 100,0 \\
\hline
\end{tabular}

Tabela 3 - Gestantes segundo tipo de sabonete e método de utilização. São Paulo, março a julho de 2008.

\begin{tabular}{lrr}
\cline { 1 - 1 } Tipo de sabo nete & $\mathbf{n}$ & $\mathbf{\%}$ \\
\hline Comum barra & 121 & 97,6 \\
Comum líquido & 2 & 1,6 \\
Outro (cas eiro) & 1 & 0,8 \\
\cline { 1 - 1 } Total & 124 & 100,0 \\
\hline Método de uso & $\mathbf{n}$ & $\mathbf{0}$ \\
\cline { 1 - 2 } Direto na pele & 82 & 66,1 \\
Emprego de buchas e esponjas & 24 & 19,4 \\
Emprego de espuma & 18 & 14,5 \\
\cline { 1 - 2 } Total & 124 & 100,0 \\
\hline
\end{tabular}

Em relação à higiene dos cabelos, constatou-se que no inverno $31(25 \%)$ gestantes relataram fazer a higiene todos os dias, 39 (31,4\%) em dias alternados, 40 (32,3\%) duas vezes por semana e $14(11,3 \%)$ uma vez na semana. No verão, os achados modificaram-se: 50 (40,3\%) gestantes lavavam o cabelo diariamente, 40 (32,3\%) em dias alternados, $28(22,6 \%)$ duas vezes na semana e seis $(4,8 \%)$ uma vez por semana. Para a totalidade das mulheres, o produto citado para a lavagem foi o xampu comum, isto é, os adquiridos em perfumarias e farmácias.

Quanto à hidratação facial, verificou-se que 57 (46\%) gestantes informaram não fazer, $38(30,6 \%)$ faziam de forma irregular e $29(23,4 \%)$ regularmente. Destas, apenas uma $(1,4 \%)$ usava produto recomendado por dermatologista; as demais $66(98,5 \%)$, usavam cosméticos de perfumarias (loções, cremes e óleos). O número de gestantes que realizava a hidratação corporal foi maior; $111(89,5 \%)$ hidratavam com produto comum, duas 
$(1,6 \%)$ com produto recomendado por dermatologista e $11(8,9 \%)$ não o faziam. Vale ressaltar que as 11 gestantes que não usavam hidratante corporal, faziam uso de sabonete com $\mathrm{pH}$ alcalino.

Em relação ao uso de protetor solar, os dados obtidos foram: $90(72,6 \%)$ gestantes afirmaram não fazer uso de protetores solar. Das $34(27,4 \%)$ mulheres que afirmaram fazer uso, $29(85,3 \%)$ passavam o produto separado de outros cosméticos e $5(14,7 \%)$ usavam o protetor conjugado à maquiagem; 12 (35,3\%) usavam diária e regularmente, 5 $(14,7 \%)$ diariamente e com reaplicação, 10 (29,4\%) de forma irregular e 7 (20,6\%) só no lazer (praia e clube). Verificou-se ainda que o uso do produto nas estações do inverno e verão é diferente; 20 (58,8\%) gestantes afirmaram não usar protetor solar durante o inverno. Os fatores de proteção solar mencionados pelas 34 gestantes são apresentados nos dados da Tabela 4 abaixo.

Tabela 4 - Uso de fator de proteção solar pelas gestantes.São Paulo, março a julho de 2008.

\begin{tabular}{lrrrr}
\hline \multirow{2}{*}{ Fator de proteção } & \multicolumn{2}{l}{ Inverno } & \multicolumn{2}{c}{ Verão } \\
\cline { 2 - 5 } & $\mathbf{n}$ & $\mathbf{\%}$ & $\mathbf{n}$ & $\mathbf{\%}$ \\
\hline Fator 15 & 6 & 42,9 & 9 & 26,5 \\
Fator 30 & 6 & 42,9 & 20 & 58,8 \\
Fator 50 & 1 & 7,1 & 3 & 8,8 \\
Não sabe & 1 & 7,1 & 2 & 5,9 \\
\hline Total & 14 & 100,0 & 34 & 100,0 \\
\hline
\end{tabular}

Sobre o controle de peso, as respostas das gestantes mostraram que 89 (71,8\%) faziam o monitoramento: dessas $49(55,1 \%)$ faziam-no mensalmente, 27 (30,3\%) semanal, $7(7,9 \%)$ quinzenal, $5(5,6 \%)$ diariamente e uma $(1,1 \%)$ fazia de forma irregular. As demais, $35(28,2 \%)$ não faziam controle; dessas 16 encontravam-se no segundo trimestre de gravidez e 19, no terceiro.

A adoção de alimentação equilibrada isto é, consumo de refeições com baixa ingesta de gordura animal e carboidratos, diversidade de frutas, verduras e leguminosas, foi investigada. Do total de gestantes, $46(37,1 \%)$ informaram manter hábito alimentar inadequado, 44 $(35,5 \%)$ mudaram a alimentação para mais saudável após a gestação e $33(26,6 \%)$ afirmaram sempre se preocupar com a qualidade da dieta: uma $(0,8 \%)$ referiu ter muitas náuseas, não conseguindo se alimentar adequadamente.

A hidratação por via oral mencionada pelas participantes indicou que $27(21,8 \%)$ ingeriam menos de um litro de líquido por dia, $69(55,6 \%)$ de um a dois litros, $17(13,7 \%)$ de dois a três litros e $11(8,9 \%)$ mais de três litros.

As gestantes foram indagadas quanto à sua percepção sobre o investimento pessoal com sua pele. A maioria, $92(74,2 \%)$, afirmou cuidar da pele. Destas $55(59,8 \%)$ afirmaram cuidar não diariamente, $31(33,7 \%)$ regularmente uma vez ao dia e a minoria, 6
$(6,5 \%)$, regularmente e mais de uma vez ao dia. Confirmaram não cuidar da pele $32(25,8 \%)$ gestantes.

A maioria (56\%) afirmou não ter recebido, da equipe de saúde, orientações sobre cuidados com a pele durante a gestação e 54 (44\%) disseram sim; destas, 32 (59,2\%) citaram o médico, $9(16,7 \%)$ a enfermeira, $8(14,8 \%)$ o médico e a enfermeira, $2(3,7 \%)$ o auxiliar de enfermagem, 1 (1,9\%) o médico, a enfermeira e o auxiliar de enfermagem e $2(3,7 \%)$ não souberam dizer qual foi o profissional.

\section{DISCUSSÃO}

As implicações dos resultados para a saúde e bemestar da gestante permeiam a discussão dos dados. Poucos estudos foram encontrados abordando as variáveis pesquisadas.

Neste estudo, $62(50 \%)$ gestantes declararam permanecer por mais de uma hora na posição em pé; $18(29 \%)$ delas por mais de seis horas ao dia. A permanência prolongada na posição em pé é mais um fator colaborador para dificultar o retorno venoso dos membros inferiores, propiciador de estase venosa, além da gestação. Se a mulher, anteriormente à gestação, já apresentava varizes os sintomas tendem a piorar após longos períodos em pé(14). Em um estudo sobre a prevalência de varizes, o autor encontrou associação com sexo feminino, lesão nas pernas e permanência na posição em pé por longo tempo ${ }^{(15)}$.

Como medida preventiva para alterações vasculares, as mulheres devem ser orientadas a não ficar em pé durante longos períodos e fazer pequenas interrupções quer com exercícios ou com elevação das pernas e pés. Quando descansarem por períodos mais longos, devem ser aconselhadas a descansar sobre seu lado esquerdo, diminuindo, assim, a pressão sobre a veia cava inferior e as veias dos membros inferiores ${ }^{(14)}$.

Em relação ao estilo de vida e prática de atividade física, os resultados vêm ao encontro de um problema do mundo moderno: o sedentarismo. Estudos epidemiológicos apontam que uma grande parcela da população não atinge as recomendações atuais quanto à prática de atividades físicas ${ }^{(16)}$.

Os benefícios das atividades para a saúde estão amplamente evidenciados, sendo vários os objetivos para a gestação. Os de interesse dermatológico são: melhorar o controle ponderal e auxiliar no retorno venoso, fundamentais para a prevenção de estrias e alterações vasculares, respectivamente.

Ginecologistas e obstetras recomendam para gestantes exercícios de intensidade moderada voltados ao período gestacional, atividades centradas nas condições de saúde e experiência em praticar exercícios, além de considerar o interesse e necessidade da mesma. As atividades que 
vêm se destacando no período gestacional, são os exercícios leves na água, caminhada e bicicleta ${ }^{(17)}$. Os resultados deste estudo sustentam esta última afirmação.

Quanto à exposição solar, os dados obtidos mostram que a maioria das gestantes esteve exposta à radiação Ultra Violeta B (UVB) em seu período mais forte e todas expostas à radiação Ultra Violeta A (UVA), variando somente o tempo de exposição. É fundamental ressaltar a importância da radiação no desencadeamento e agravamento de manchas. Estudo recente que investigou a percepção da gestante sobre as mudanças ocorridas na pele, durante o período gestacional, encontrou a mancha como a alteração mais citada ${ }^{(18)}$.

É sabido que a luz UVB é mais carcinogênica, não incide em níveis homogêneos durante todo o dia, é mais forte entre 10:00 h e 15:00 h e a luz UVA é estimuladora de melanina, responsável pelas alterações pigmentares; consegue ultrapassar a camada de ozônio praticamente durante o dia inteiro, persiste no inverno e em dias nublados, e é capaz de ultrapassar vidros, inclusive com películas protetoras $^{(12,19)}$.

A Sociedade Brasileira de Dermatologia recomenda que todas as medidas de proteção sejam adotadas quando houver exposição ao sol em razão da ação cumulativa dos raios solares: uso de chapéu, camisetas e protetor solar. As barracas de praia devem ser de algodão ou lona, porque esses tecidos absorvem 59\% da radiação ultravioleta ${ }^{(19)}$.

Sobre a higiene corporal os dados mostraram que a maior parte das mulheres tomava mais de um banho ao dia: banhos quentes e demorados, usava sabonete comum, em barra, diretamente sobre a pele e com $\mathrm{pH}$ alcalino. Todas essas práticas são desfavoráveis à pele.

A frequência elevada do banho pode ressecar a pele. Este sinal pode ser identificado por meio de descamação, vermelhidão, esfoliação e rachaduras. Indica-se avaliar o grau de ressecamento para determinar se os banhos frequentes são benéficos ${ }^{(20)}$. Existem divergências a este respeito, alguns especialistas afirmam que a água não faz mal à pele, e sim o uso excessivo de sabonetes. $\mathrm{O}$ tema carece de evidências.

Os banhos quentes e demorados não são recomendados por reduzirem o manto lipídico. $\mathrm{O}$ uso de água muito quente ou quente pode contribuir para agravar e tornar a pele mais suscetível a agravos. O calor é um fator físico que contribui para os efeitos dos agentes químicos, como os sabonetes ${ }^{(21)}$.

Nos casos de mais de um banho ao dia, a indicação é limitar o uso de sabonetes; ensaboar o corpo todo em apenas um dos banhos e nos demais ensaboar apenas axilas, genitais, regiões inguinais e pés ${ }^{(12)}$.

$\mathrm{O}$ emprego repetido dos sabonetes pode alterar o $\mathrm{pH}$ da superfície cutânea ${ }^{(22)}$. O banho com sabonete desencadeia um aumento no $\mathrm{pH}$ da pele que interfere na proteção fisiológica, manto ácido, provocando mudança na composição da flora bacteriana cutânea e na atividade das enzimas da epiderme. Outra consequência é a dissolução da gordura da superfície da epiderme que influencia nas condições de hidratação e predispõe à secura e à descamação da pele ${ }^{(23)}$. $\mathrm{O} \mathrm{pH}$ alcalino é o principal responsável pelo potencial irritante e desidratante da pele, além de propiciar o aumento do Propionibacterim acnes ${ }^{(24)}$.

Um estudo avaliou o $\mathrm{pH}$ de 42 sabonetes destinados ao uso adulto, disponíveis no mercado brasileiro, incluindo apresentações em barra e em líquido. A maioria dos produtos em barra apresentou $\mathrm{pH}$ entre 9 e 10 e os líquidos $\mathrm{pH}$ menor do que $8^{(25)}$. A literatura preconiza o uso de sabonetes com $\mathrm{pH}$ ácido, uma vez que não interferem tão intensamente na microflora cutânea e possuem menor potencial deletério, pois se aproximam do $\mathrm{pH}$ fisiológico que varia entre 4,2 a 5,9, dependendo da área do corpo aferida. Não foram encontrados estudos sobre o uso de buchas e esponjas; alguns dermatologistas reconhecem que a bucha também pode acabar com a suavidade da pele, quando usada diariamente. É uma alternativa para limpar áreas mais ásperas e escuras como joelhos, cotovelos, pés e axilas. O problema é a fácil contaminação desses materiais.

Quanto à higiene dos cabelos, verificou-se neste estudo que a maioria das gestantes realizava a limpeza com frequência, sendo maior no verão. Não foram encontrados estudos sobre esta temática. Segundo os dermatologistas, a lavagem diária dos cabelos não interfere com os bulbos capilares e o produto utilizado deve ser selecionado com critério, deve ser seguro e não contribuir para a oleosidade da pele.

Em relação à hidratação facial e corporal, os resultados mostraram que a maioria das gestantes fazia hidratação: 67 (54\%) facial e $113(91,1 \%)$ corporal. A maior frequência da hidratação corporal pode ser compreendida em decorrência do crescimento abdominal, o risco de estrias e a veiculação de informações provenientes da mídia. Estes aspectos não foram explorados neste estudo.

$\mathrm{O}$ uso de cosméticos hidratantes constitui uma das mais importantes classes de produtos para preservar o manto hidrolipídico da pele, pois melhoram a condição da pele, aproximando-a de suas condições ideais, pois aumentam a quantidade de água no estrato córneo ${ }^{(26)}$. A pele seca além de poder gerar desconforto, como decorrência da alteração do aspecto visual e sensorial da pele, também contribui para o surgimento de características como descamação, microfissuras e sangramentos ${ }^{(27)}$.

A utilização inadequada dos produtos disponíveis no mercado pode contribuir para o surgimento de problemas como acne e dermatite seborreica. É fundamental considerar que a gestante tem uma pele com maior fluxo sanguíneo e com poder de absorção 
maior. Especialistas recomendam produtos com menores riscos de alergia e absorção, que não contenham corantes e possuam fragrâncias suaves para proporcionar maior segurança.

A avaliação dos parâmetros reológicos, isto é, viscosidade do produto, ponto de fluidez, espalhabilidade e estabilidade físico-química, como fatores de seleção entre emulsões cosméticas semelhantes é de muita importância para se obter efeitos satisfatórios ${ }^{(28)}$. Neste estudo, apenas três gestantes usaram produtos indicados por especialistas.

A boa hidratação da pele durante a gestação tem sido uma medida para a prevenção de estrias, ainda que não existam fórmulas milagrosas para evitá-las ${ }^{(2)}$.

Os dados obtidos sobre o uso de protetor solar são preocupantes. A maioria, 90 (72,6\%) gestantes, não fazia uso de protetor, uma minoria, $17(13,7 \%)$ faz uso regularmente e apenas 5 (4\%) faziam a reaplicação. Quanto ao fator de proteção, chama à atenção a baixíssima citação de uso no inverno e o desconhecimento do fator utilizado citado por algumas.

O sol associado à fotossensibilização decorrente das alterações hormonais na gestação torna imprescindível a indicação do uso de protetor solar. Pesquisadores demonstraram, em ensaio clínico de 12 meses, a eficácia do protetor solar de amplo espectro na prevenção do melasma em gestantes. Das 185 mulheres que completaram o estudo, apenas cinco novos casos foram constatados, uma ocorrência de $2,7 \%$, dado inferior aos $53 \%$ anteriormente observados em outro estudo com população não protegida. Doze gestantes iniciaram o estudo com o melasma e oito obtiveram parcial clareamento das manchas com o uso do protetor ${ }^{(29)}$. Outras pesquisas também confirmam o papel positivo da proteção solar na prevenção e tratamento do melasma. Todos os tipos de pele, em maior ou menor escala, estão sujeitos a alterações, por isso, precisam de uma proteção efetiva.

Existem no mercado produtos com fatores de proteção solar (FPS) variáveis. A partir do FPS 20, que protege a pele cerca de $95 \%$ dos raios UVB, os benefícios aumentam muito pouco e mesmo o mais alto índice não consegue bloquear totalmente a penetração dos raios UVB na epiderme. Os dermatologistas costumam recomendar FPS de 15 a 30, mais do que isso é muita química para pouca proteção ${ }^{(19)}$. Para atividades ao ar livre como praia, piscinas, clubes o fator deve ser aumentado ${ }^{(12)}$.

Os dermatologistas recomendam protetores com nível de proteção declarado para UVA e UVB; veículo adequado para a pele da gestante, menos comedogênicos, com menos substâncias químicas, menos alérgenos (corantes ou fragrâncias que possam irritar a pele) e que tenham eficácia de proteção contra eritema e pigmentação. A reaplicação é necessária a cada duas horas ou após imersão em água ${ }^{(12,19)}$. Se as pessoas aplicassem os protetores solares de maneira uniforme e adequada não haveria necessidade de fatores de proteção solar superior a 15. A espessura de aplicação acordada internacionalmente é de $2 \mathrm{mg} / \mathrm{cm}^{2}$. Estudos têm mostrado que os consumidores aplicam-se normalmente entre 0,5 a $1,3 \mathrm{mg} / \mathrm{cm}^{2}$ menos que o recomendado ${ }^{(30)}$.

Em relação ao controle de peso, neste estudo encontrou-se, número significativo de gestantes, 35 $(28,2 \%)$ que não fizeram controle de peso até a data da coleta, inclusive no $3^{\circ}$ trimestre de gestação.

Do ponto de vista dermatológico, o controle do peso é fundamental para a prevenção de estrias. Profissionais de saúde recomendam e mulheres têm usado óleos e suplementos vitamínicos para prevenir estrias gravídicas, entretanto, não há grandes ensaios clínicos, prospectivos que mostrem resultados animadores. As terapias, definitivamente, não provaram redução significativa do desenvolvimento de estrias ${ }^{(3-4,6)}$. É consenso que a gestante deve seguir as recomendações obstétricas, para que não haja um aumento significativo e abrupto de peso durante os diversos períodos gestatórios, em especial, no último trimestre; esta é a medida principal na prevenção de estrias ${ }^{(9)}$.

Quanto à alimentação e hidratação, os dados apontam que $46(37 \%)$ declararam ter hábito alimentar inadequado e, apenas $28(22,5 \%)$ das gestantes ingeriam mais de dois litros de líquido por dia. A inadequação alimentar representa risco para ganho de peso e estrias, e a baixa ingesta hídrica representa risco para ressecamento da pele.

O consumo energético é um importante determinante do ganho de peso durante a gestação que, por sua vez, é um dos fatores que sistematicamente vem apresentando associação positiva com a retenção de peso pós-parto e obesidade materna ${ }^{(31)}$. O consumo de água é altamente recomendado, salvo as restrições hídricas, pois, além de não conter nenhuma caloria, melhora o funcionamento do organismo e hidrata a pele ${ }^{(19)}$.

O fato de $70(56 \%)$ gestantes afirmarem não terem recebido qualquer orientação sobre cuidados com a pele é de causar inquietação. O conhecimento possibilita melhores condições para as pessoas tomarem decisões conscientes e responsáveis Chama a atenção a baixa participação das enfermeiras no processo educativo, tendo em vista o cuidar como o cerne da profissão.

É pertinente refletir sobre a importância e necessidade de sensibilização e treinamento da equipe de saúde sobre os problemas considerados de baixa morbidade. Não há dúvidas de que há subvalorizaçao das alterações cutâneas enfrentadas pelas gestantes ${ }^{(4)}$.

\section{CONCLUSÕES}

Os resultados evidenciaram a importância da inclusão da abordagem sobre cuidados com a pele nos 
programas de educação em saúde dos serviços que proporcionam atendimento à gestante. A equipe de saúde pautada nos princípios de prevenção de agravos deve comprometer-se em manter ou maximizar o nível de bem-estar das gestantes, atendendo às necessidades de

\section{REFERÊNCIAS}

1. Lawley TJ, Yancey KB. Skin changes and diseases in pregnancy. In: Freedberg IM, Eisen AZ, Wolff K, Austen F, Goldsmith LA, Katz SI, editors. Fitzpatrick's dermatology in general medicine. 6th ed. New York: McGraw-Hill; 2003. p.1361-6.

2. Mandelbaum SH. Dermatologia na gestante. Cap.31. In: Cucé LC, Festa Neto C. Manual de dermatologia. 2a ed. São Paulo: Atheneu; 2001. p. 549-53.

3. Barankin B, Silver SG, Carruthers A. The skin in pregnancy. J Cutan Med Surg. 2002;6(3):236-40.

4. Alves GF, Varella TCN, Nogueira LSC. Dermatologia e gestação. An Bras Dermatol. 2005;80(2):179-86.

5. Vergnanini AL. Dermatopatias. In: Neme B. Obstetrícia básica. 3a ed. São Paulo: Sarvier; 2006. Cap. 60.

6. Nussbaum R, Benedetto AV. Cosmetic aspects of pregnancy. Clin Dermatol. 2006;24(2):133-41.

7. Elling SV, Powell FC. Physiological changes in the skin during pregnancy. Clin Dermatol. 1997;15(1):35-43. Review.

8. Muzaffar F, Hussain I, Haroon TS. Physiologic skin changes during pregnancy: a study of 140 cases. Int J Dermatol. 1998;37(6):429-31.

9. Brasil. Sociedade Brasileira de Dermatologia - SBD. Censo 2006. Rio de Janeiro (RJ). Disponível em: http:// www.sbd.org.br.

10. Oliveira MS, Muller MC, Moraes JFD, Ludwig MWB. Qualidade de vida e localização da lesão em pacientes dermatológicos. An Bras Dermatol. 2009;84(2):143-50.

11. Taborda ML, Weber MB, Teixeira KAM, Welter EQ. Avaliação da qualidade de vida e do sofrimento psíquico de pacientes com diferentes dermatoses em um centro de referência em dermatologia no sul do país. An Bras Dermatol. 2010;85(1):52-6.

12. Reis VMS, Patriarca M. Prevenção das alterações da pele na gestação [Internet]. São Paulo: Organon Farmacêutica. [citado 2008 Ago 23]. Disponível em: <http:// www.pelenagravidez.com.br>.

13. Honein MA, Paulozzi LJ, Erickson JD. Continued occurrence of Accutane-exposed pregnancies. Teratology. 2001;64(3):142-7.

14. Bamigboye AA, Smyth R. Interventions for varicose veins and leg oedema in pregnancy. Cochrane Database Syst Rev. 2007;(1):CD001066. Review.

15. Jawien A. The influence of environmental factors in chronic venous insufficiency. Angiology. 2003;54 Suppl 1:S19-31. Review.

16. Hallal PC, Dumith SC, Bastos JP, Reichert FS, Siqueira FV, Azevedo MR. Evolução da pesquisa epidemiológica em atividade física no Brasil: revisão sistemática. Rev Saúde saúde e evitando tratamentos posteriores mais intervencionistas. Os achados desta primeira exploração dão subsídios para o planejamento de ações mais plenas, possibilitando um cuidar eficaz para a mulher no ciclo gravídico-puerperal.

Pública = J Public Health. 2007;41(3):453-60.

17. Batista DC, Chiara VL, Gugelmin SA, Martins PD. Atividade física e gestação: saúde da gestante não atleta e crescimento fetal. Rev Bras Saúde Matern Infant. 2003;3(2):151-8.

18. Urasaki MBM. Alterações fisiológicas da pele percebidas por gestantes assistidas em serviços públicos de saúde. Acta Paul Enferm. 2010;23(4):519-25.

19. Macedo OR. Segredos da boa pele: preservação e correção. 2a ed. São Paulo: Senac; 2001.

20. Potter PA, Perry AG. Fundamentos de enfermagem. Rio de Janeiro: Elsevier; 2005. Cap. 38 Higienização.

21. Pimentel MIF, Matta VF. Dermatoses ocupacionais de contato. An Bras Dermatol. 1998;73(4):361-5.

22. Korting HC, Braun-Falco O. The effect of detergents on skin $\mathrm{pH}$ and its consequences. Clin Dermatol. 1996;14(1):23-7.

23. Gfatter R, Hackl P, Braun F. Effects of soap and detergents on skin surface $\mathrm{pH}$, stratum corneum hydration and fat content in infants. Dermatology. 1997;195(3):258-62.

24. Schmid MH, Korting HC. The concept of the acid mantle of the skin: its relevance for the choice of skin cleansers. Dermatology. 1995;191(4):276-80.

25. Volochtchuk OM, Fujita EM, Fadel APC, Auada MP, Almeida T, Marinoni LP. Variações do pH dos sabonetes e indicações para sua utilização na pele normal e na pele doente. An Bras Dermatol. 2000;75 (6):697-703.

26. Leonardi GR, GasparLR, Campos PMBGM. Estudo da variação do $\mathrm{pH}$ da pele humana exposta à formulação cosmética acrescida ou não das vitaminas A, E ou de ceramida por metodologia não invasiva. An Bras Dermatol. 2002;77 (5):563-9.

27. Lodén M. The clinical benefit of moisturizers. J Eur Acad Dermatol Venereol. 2005;19(6):672-88; quis 686-7.

28. Milan ALK, Milão D, Souto AA, Corte TWF. Estudo da hidratação da pele por emulsões cosméticas para xerose e sua estabilidade por reologia. Rev Bras Ciênc Farm. 2007;43 (4):649-57.

29. Lakhadar H, Zouhair K, Khadir K, Essari A, Richard A, Seité $\mathrm{S}$, Rougier A. Evaluation of the effectiveness of a broadspectrum sunscreen in the prevention of chloasma in pregnant women. J Eur Acad Dermatol Venereol. 2007;21(6):738-42.

30. Diffey B. Has the sun protection factor had its day? BMJ. 2000;320(7228):176-7. Comment in: BMJ. 2000;320(7244):1274-5. BMJ. 2000;320(7244):1275. BMJ. 2002;324(7352):1526.

31. Lacerda EMA, Leal MC. Fatores associados com a retenção e o ganho de peso pós-parto: uma revisão sistemática. Rev Bras Epidemol. 2004;7(2):187-200. 